\title{
A mixed formulation of mortar-based contact with friction
}

\author{
I. Temizer* \\ Department of Mechanical Engineering, Bilkent University, 06800 Ankara, Turkey
}

\section{A R T I C L E I N F O}

\section{Article history:}

Received 9 April 2012

Received in revised form 1 December 2012

Accepted 4 December 2012

Available online 12 December 2012

\section{Keywords:}

Contact

Friction

Mortar method

Mixed formulation

Large deformation

\begin{abstract}
A B S T R A C T
A classical three-field mixed variational formulation of frictionless contact is extended to the frictional regime. The construction of the variational framework with respect to a curvilinear coordinate system naturally induces projected mortar counterparts of tangential kinetic and kinematic quantities while automatically satisfying incremental objectivity of the associated discrete penalty-regularized mortar constraints. Mixed contact variables that contribute to the boundary value problem are then obtained through unconstrained, lumped or constrained recovery approaches, complemented by Uzawa augmentations. Patch tests and surface locking studies are presented together with local and global quality monitors of the contact interactions in two- and three-dimensional settings at the infinitesimal and finite deformation regimes.
\end{abstract}

(c) 2012 Elsevier B.V. All rights reserved.

\section{Introduction}

The incorporation of friction into contact algorithms requires additional effort, in particular to ensure an objective integration of the evolution laws that describe irreversible interface mechanics but also in obtaining the associated algorithmic linearization to achieve an asymptotically quadratic convergence. The consideration of friction in the context of mortar approaches has developed in parallel with the frictionless case. Although the mortar discretization of the kinetic and kinematic contact variables are similar to the frictionless case, the description of the projected tangential kinematics is typically realized in terms of vector quantities and many studies are still limited to a two-dimensional setting. A vectorial description requires a careful construction of the update scheme for the tractions while the procedure is well-established in the continuum setting where the components with respect to a local curvilinear coordinate system are conveniently employed. Accordingly, contrary to normal contact, the way in which the tangential constraints are treated differs considerably in mortar approaches. In [1], the three-field mixed variational formulation foundation for mortar-based frictionless contact treatment was investigated. One goal of the present work is to construct a unified mixed variational treatment of normal and tangential contact constraints by extending the original idea of [2] for frictionless contact. The advantage of such a unification is that the algorithmic aspects of the complete tangential formulation emanate directly from a three-field statement, the only exception being the necessity of

\footnotetext{
* Tel.: +90 (312) 2903064 .

E-mail address: temizer@bilkent.edu.tr
}

an independent mortar projection of the slip criterion. The advocated method lacks an intermediate surface to ensure a highly accurate numerical integration but it has all the remaining major ingredients of a mortar-based approach. Moreover, it offers alternatives to popular mortar schemes and provides numerical demonstrations of their viability. It is highlighted that a large class of algorithms associated with the classical node-to-segment approach and its variants are omitted from the present discussion where emphasis is strictly on mortar methods. The reader is referred to $[3,4]$ for extensive references on alternative approaches, to [5] for a recent survey of numerical algorithms for contact problems.

Starting with applications in small deformation contact problems [6,7], it was shown that mortar methods satisfy the patch test while avoiding surface locking [8-10]. Additional investigations in the finite deformation regime with large sliding have further demonstrated the ability of the mortar method to successfully address problems that proved to be problematic for the classical node-tosegment schemes [11-18]. The incorporation of friction in mortar approaches goes back to the mathematical analysis of [19]. A finite element framework was subsequently investigated in [20] within a kinematically linearized two-dimensional framework. Based on parallel developments in domain decomposition [21], an extension to three-dimensional large deformation contact was introduced in [22]. Here, particular emphasis was given to the segmentation of the contact interface for an accurate evaluation of the contact integrals. Additionally, the construction of an incrementally objective slip definition was presented. Further investigations in a twodimensional setting were presented in [23]. Two-dimensional studies on the possibilities for quadratic elements and integration 
without an intermediate surface were explored in [24]-see also [25] for three-dimensional implementations for quadratic elements with an intermediate surface. While the mentioned works incorporated a regularization of the normal and tangential constraints through a penalty method, a Lagrange multiplier approach was introduced in [26] in a two-dimensional setting and a threedimensional framework with dual Lagrange multipliers was presented in [27]-see also [28] for two-dimensional investigations. Recent studies also introduced efficient semi-smooth Newton approaches, based on [29,30], for two-dimensional mechanical [31] and three-dimensional thermomechanical [32] problems. See $[33,34]$ for further three-dimensional examples.

In order to construct a three-dimensional mixed formulation of mortar-based contact treatment with friction for large deformations, the three-field mixed variational formulation of [2] is extended to the tangential kinematics in Section 2 on the basis of the discussion in [1]. Starting from the continuum setting, a direct formulation with respect to a curvilinear coordinate system automatically ensures the objectivity of the stick and slip formulations. Constrained and lumped recovery approaches for the tangential interactions are developed and subsequently linked with an unconstrained formulation. Consistency statements with respect to Uzawa augmentations are discussed and subsequently the algorithmic linearization of the overall algorithm is presented in Section 3. Numerical investigations are presented in Section 4 where, in addition to comparing the local traction quality with analytical solutions, patch tests for tied non-flat surfaces as well as examples with significant evolutions of the contact interface are discussed.

Throughout the theoretical developments and numerical investigations, various details and observations have been omitted together with related references in order to minimize overlap with the presentation in [1]. In particular, the notation introduced therein is employed without restating definitions, i.e. the presentation is not entirely self-contained. However, a repetition of critical aspects has been incorporated to a minor extent.

\section{Three-field mixed tangential contact treatment}

\subsection{Continuum formulation}

The contact contribution to the weak form can be expressed as

$\delta \mathcal{G}^{c}:=\int_{\partial \mathcal{R}_{0}^{c}}(\delta \boldsymbol{x}-\delta \boldsymbol{y}) \cdot \boldsymbol{p} d A=-\int_{\partial \mathcal{R}_{0}^{c}}\left(\delta g_{N} p_{N}+\delta \xi^{\alpha} \tau_{\alpha}\right) d A$,

under the standard assumption of an exact satisfaction of the impenetrability condition $g_{N}=0$ to simplify the tangential contribution, with $\tau_{\alpha}$ as the covariant components of the tangential traction $[3,4]$. Here, the convention is such that on the deformed configuration of the master surface the convected curvilinear coordinates $\xi^{\alpha}$ induce the covariant basis vectors $\boldsymbol{a}_{\alpha}:=\frac{\partial \boldsymbol{y}}{\partial \xi^{\alpha}}$ which define the covariant metric components $a_{\alpha \beta}:=\boldsymbol{a}_{\alpha} \cdot \boldsymbol{a}_{\beta}$. The inverse (contravariant metric) components $a^{\alpha \beta}$, with $a^{\alpha \gamma} a_{\gamma \beta}=\delta_{\beta}^{\alpha}$ as the Kronecker delta, then define the contravariant basis vectors $\boldsymbol{a}^{\alpha}:=a^{\alpha \beta} \boldsymbol{a}_{\beta}$ such that a generic vector $\boldsymbol{v}$ in the tangent space admits the representations $\boldsymbol{v}=v^{\alpha} \boldsymbol{a}_{\alpha}=v_{\alpha} \boldsymbol{a}^{\alpha}$.

Within a time/load-discretized setting with step index $n$, the incremental updates

$g_{T}^{\alpha}:=\xi^{\alpha}-\xi^{\alpha, n}, \quad p_{T \alpha}:=\tau_{\alpha}-\tau_{\alpha}^{n}$

are of interest where variables without a time/load index belong to $n+1$. For tangential contact, variational terms are identified in terms of the history variables and the incremental updates as $\delta \mathcal{G}_{T}^{c}=-\int_{\partial \mathcal{R}_{0}^{c}} \delta g_{T}^{\alpha}\left(\tau_{\alpha}^{n}+p_{T \alpha}\right) d A$

Here, the Coulomb slip criterion

$\sigma\left(\tau, p_{N}\right):=\|\tau\|-\mu p_{N} \leqslant 0$

is assumed where $\mu$ is the (constant) friction coefficient and $\|\tau\|^{2}=\tau_{\alpha} a^{\alpha \beta} \tau_{\beta}$. During slip, the evolution of the projection coordinates is obtained from the objective statement

$s_{\alpha}:=\frac{\tau_{\alpha}}{\|\tau\|} \rightarrow \dot{g}_{T}^{\alpha}=\dot{\lambda} \frac{\partial \sigma}{\partial \tau_{\alpha}}=\dot{\lambda} a^{\alpha \beta} s_{\beta}$,

where $\lambda$ is the consistency parameter, with which the tangential constraints can be stated as

$\sigma \leqslant 0, \quad \dot{\lambda} \geqslant 0, \quad \sigma \dot{\lambda}=0$.

\subsection{Mixed formulation}

Within a penalty regularization of the contact constraints, the tangential contact contribution to the weak form emanates, the latter assuming the stick state, from the variation of

$\mathcal{G}_{T}^{c}=-\int_{\partial \mathcal{R}_{0}^{c}}\left(g_{T}^{\alpha} \tau_{\alpha}^{n}+\frac{\epsilon_{T}}{2} g_{T}^{\alpha} a_{\alpha \beta} g_{T}^{\beta}\right) d A$,

such that $p_{T \alpha}=\epsilon_{T} a_{\alpha \beta} g_{T}^{\beta}$. Algorithmically, the continuum formulation leads to the update

$\tau_{\alpha}=\tau_{\alpha}^{n}+\epsilon_{T}\left(a_{\alpha \beta} g_{T}^{\beta}-\Lambda s_{\alpha}\right)$,

where $\Lambda$, the time-discrete version of $\dot{\lambda}$, vanishes in the case of stick. In obtaining this update, the variation of $a_{\alpha \beta}$ is omitted from the weak form-see Section 3 for a discussion.

The approach of [2] for classical three-field mixed formulation of normal contact is now extended to tangential contact. The initial steps largely follow [1] and are only briefly addressed. Key difficulties associated with the kinetic quantities will be treated in detail. For this purpose, introducing mixed tangential kinematic variables $\gamma_{T}^{\alpha}$, the following three-field mixed formulation in terms of $\left\{g_{T}^{\alpha}, \gamma_{T}^{\alpha}, p_{T \alpha}\right\}$ is introduced:

$$
\begin{aligned}
\mathcal{C}_{T}\left[g_{T}^{\alpha}, \gamma_{T}^{\alpha}, p_{T \alpha}\right]:= & -\frac{\epsilon_{T}}{2} \int_{\partial \mathcal{R}_{o}^{c}} \gamma_{T}^{\alpha} a_{\alpha \beta} \gamma_{T}^{\beta} d A+\int_{\partial \mathcal{R}_{o}^{c}} p_{T \alpha}\left(\gamma_{T}^{\alpha}-g_{T}^{\alpha}\right) d A \\
& -\int_{\partial \mathcal{R}_{o}^{c}} g_{T}^{\alpha} \tau_{\alpha}^{n} d A .
\end{aligned}
$$

In this section, a trial stick stage is intrinsically assumed but not explicitly denoted for notational brevity. Unlike the normal part, the covariant metric must appear as an additional purely geometrical term in this functional. The variation of $\mathcal{C}_{T}$ delivers the tangential contribution $\delta \mathcal{G}_{T}^{c}$ to the linear momentum balance as well as the equalities

$\int_{\partial \mathcal{R}_{o}^{c}} \delta p_{T \alpha} \gamma_{T}^{\alpha} d A=\int_{\partial \mathcal{R}_{o}^{c}} \delta p_{T \alpha} g_{T}^{\alpha} d A$

and

$\int_{\partial \mathcal{R}_{0}^{c}} \delta \gamma_{T}^{\alpha} p_{T \alpha} d A=\epsilon_{T} \int_{\partial \mathcal{R}_{0}^{c}} \delta \gamma_{T}^{\alpha} a_{\alpha \beta} \gamma_{T}^{\beta} d A$

In order to complement the normal formulation in the mortar setting, the tangential part needs to be defined in terms of mortar projections to the nodes. This is realized by admitting discretizations of the mixed variables which are inherited from the slave surface discretization via $[11,22]$

$p_{T \alpha}=\sum_{I} N^{I} p_{T \alpha}^{I}, \quad \gamma_{T}^{\alpha}=\sum_{I} N^{I} \gamma_{T}^{\alpha, I}$. 
Now, Eq. (2.10) can be rewritten as

$$
\left\langle\sum_{I} \delta p_{T \alpha}^{I} N^{I} \gamma_{T}^{\alpha}\right\rangle=\left\langle\sum_{I} \delta p_{T \alpha}^{I} N^{I} g_{T}^{\alpha}\right\rangle,
$$

such that due to the arbitrariness of $\delta p_{T \alpha}^{I}$ it implies, together with an explicit enforcement of the active set,

$\bar{\gamma}_{T}^{\alpha, I}=\chi^{I} \bar{g}_{T}^{\alpha, I}$.

Clearly, $\gamma_{T}^{\alpha}$ cannot be pointwise equal to $g_{T}^{\alpha}$ in general since the former inherits the discretization of the slave surface only whereas the latter varies according to the discretizations of both the slave and the master surfaces. In order to recover the kinematic mixed mortar quantity $\gamma_{T}^{\alpha, I}$, it is set to zero for all inactive points. This choice is designated the default approach and will be referred to as the constrained recovery procedure. The final result is

$\gamma_{T}^{\alpha, I}=\sum_{J} \widehat{\Psi}^{I J} \bar{\gamma}_{T}^{\alpha_{J} J}=\sum_{J} \widehat{\Psi}^{I J} \chi^{J} \bar{g}_{T}^{\alpha, J}$.

It is highlighted again that the choice of vanishing $\gamma_{T}^{\alpha, I}$ for $I \notin \mathcal{A}$ is enforced explicitly here.

The treatments of normal and tangential parts deviate in the definition of the kinetic quantities. Eq. (2.11) can be rewritten as

$\left\langle\sum_{I} \delta \gamma_{T}^{\alpha, I} N^{I} p_{T \alpha}\right\rangle=\left\langle\sum_{I} \delta \gamma_{T}^{\alpha, I} N^{I} a_{\alpha \beta} \gamma_{T}^{\alpha}\right\rangle$

In view of the arbitrariness of the variations $\delta \gamma_{T}^{\alpha, I}$, this implies for $I \in \mathcal{A}$

$\bar{p}_{T \alpha}^{I}=\epsilon_{T} \sum_{J} \bar{a}_{\alpha \beta}^{I J} \gamma_{T}^{\beta, J}$,

where

$\bar{a}_{\alpha \beta}^{I J}:=\left\langle N^{I} a_{\alpha \beta} N^{J}\right\rangle$

is the projected metric. $\bar{a}_{\alpha \beta}^{I J}$ has element-level connectivity and therefore coupling among $\gamma_{T}^{\beta, J}$ does not lead to significant additional computational cost. $\bar{p}_{T \alpha}^{I}$ is explicitly set to zero for $I \notin \mathcal{A}$ since while $\bar{\gamma}_{T}^{\alpha, I}=0$ is enforced at these points (2.17) does not ensure $\bar{p}_{T \alpha}^{I}=0$ in general. This is in contrast with the normal contribution where $\bar{\gamma}_{N}^{I}=0$ implies $\bar{p}_{N}^{I}=0$ for $I \notin \mathcal{A}$.

Clearly, due to the presence of the metric in (2.11), a local relation between the mixed quantities $p_{T \alpha}^{I}$ and $\gamma_{T}^{\alpha, I}$ cannot be established-cf. (2.38). Rather, the former must be recovered from its projected counterpart. In this recovery, similar to $\gamma_{T}^{\alpha, I}, p_{T \alpha}^{I}$ is set to zero for all inactive nodes. This delivers a result similar to (2.15):

$p_{T \alpha}^{I}=\sum_{J} \widehat{\Psi}^{I J} \chi^{I} \bar{p}_{T \alpha}^{J}$

Here, $\chi^{J}$ is retained to highlight that the projected tangential traction is also defined only for active nodes.

Provided all nodes remain in a state of stick, this completes the determination of the terms which are necessary for the evaluation of the tangential contribution to the weak form of the linear momentum balance. The approach summarized for this part also forms the basis for slip check in the mortar setting, which is treated in the next section.

\subsection{Tangential constraints}

The slip surface expression which defines the tangential constraints must be proposed independently of the mixed formulation. Since the projected pressure $\bar{p}_{N}^{I}$ but not its mixed counterpart $p_{N}^{I}$ is guaranteed to be non-negative for $I \in \mathcal{A}$, check for stick-slip status should be carried out using the projected tangential tractions that are bounded by $\mu \bar{p}_{N}^{I}>0$ in magnitude.
Algorithmically, the computed projected tangential tractions from Section 2.2 are trial updates such that

$\tau_{\alpha}^{I, t r}=\tau_{\alpha}^{I, n}+p_{T \alpha}^{I}, \quad \bar{\tau}_{\alpha}^{I, t r}=\bar{\tau}_{\alpha}^{I, n}+\bar{p}_{T \alpha}^{I}$,

where the first equality, although not used in the evaluation of the slip surface, is stated for future reference in Section 2.4. Here, the discretization

$\tau_{\alpha}=\sum_{I} N^{I} \tau_{\alpha}^{I}$

and the associated projections of the history variables are naturally induced by the update scheme.

To check for slip, a norm $\left\|\boldsymbol{v}^{I}\right\|$ needs to be introduced using a discrete metric $m^{\alpha \beta, I}$ :

$\left\|\boldsymbol{v}^{I}\right\|^{2}:=v_{\alpha}^{I} m^{\alpha \beta, I} v_{\beta}^{I}$

The expression for the slip criterion now takes the discrete form

$\bar{\sigma}^{I, t r}=\left\|\bar{\tau}^{I, t r}\right\|-\mu \bar{p}_{N}^{I}:\left\{\begin{array}{l}\text { if } \leqslant 0 \text { then Stick, } \\ \text { if }>0 \text { then Slip. }\end{array}\right.$

It is highlighted that $\overrightarrow{\vec{\tau}}^{I, t r}$ is introduced only for notational convenience. An explicit definition for this quantity is not needed. Now, the discrete slip criterion highlights the two main requirements from the definition of $m^{\alpha \beta, I}$. It should (i) represent the identity for a Cartesian coordinate system and (ii) yield a norm that is dimensionally consistent with the projected pressure. Since $\bar{a}_{\alpha \beta}^{I I}$ is already defined, let $\bar{a}^{\alpha \beta, I I}$ represent the components of its inverse. ${ }^{1} \bar{a}^{\alpha \beta, I I}$ does not satisfy the two requirements but if it is scaled by its value at an identity metric to define

$m_{\alpha \beta}^{I}=\frac{\bar{a}_{\alpha \beta}^{I I}}{\Phi^{I I}}$,

then the components of its inverse

$m^{\alpha \beta, I}=\Phi^{I I} \bar{a}^{\alpha \beta, I I}$,

satisfy the requirements.

When violated, the slip criterion delivers the discrete counterpart

$\bar{\tau}_{\alpha}^{I}=\mu \bar{p}_{N}^{I} s_{\alpha}^{I}$

of the classical update, where the slip direction

$s_{\alpha}^{I}=\frac{\bar{\tau}_{\alpha}^{I, t r}}{\left\|\bar{\tau}^{I, t r}\right\|}=m_{\alpha \beta}^{I} \frac{\partial \bar{\sigma}^{I, t r}}{\partial \bar{\tau}_{\beta}^{I, t r}}$

is introduced. The overall frictional update may also be stated in a form similar to the continuous version (2.8):

$\bar{\tau}_{\alpha}^{I}=\bar{\tau}_{\alpha}^{I, n}+\epsilon_{T}\left(\sum_{J} \bar{a}_{\alpha \beta}^{I J} \gamma_{T}^{\beta, J}-\bar{\Lambda}^{I} s_{\alpha}^{I}\right)$

Consequently, the discrete versions of the tangential constraints are $\bar{\sigma}^{I} \leqslant 0, \quad \bar{\Lambda}^{I} \geqslant 0, \quad \bar{\sigma}^{I} \bar{\Lambda}^{I}=0$.

In (2.28), it is clearly seen that while an element-level connectivity arises in the trial update tractions the locality of the stick/slip check is preserved, which is computationally advantageous. Following the update (2.28) for all active nodes, the mixed tractions are recovered by solving the system of equations emanating from (2.21):

$\tau_{\alpha}^{I}=\sum_{J} \widehat{\Psi}^{I J} \chi^{J} \bar{\tau}_{\alpha}^{J}$

${ }^{1}$ Explicitly, $\bar{a}^{\alpha \gamma, I I} \bar{a}_{\gamma \beta}^{I I}=\delta_{\beta}^{\alpha}$ as an exception to the projection notation: $\bar{a}^{\alpha \gamma, I I} \neq\left\langle N^{I} a^{\alpha \gamma} N^{I}\right\rangle$. 
Consequently, the slip criterion is not necessarily satisfied in terms of the mixed tractions and pressure.

While the stick formulation steps were directly induced by the mixed formulation without ambiguity, the slip formulation allows for some flexibility in definitions within the present setting, in particular for $m^{\alpha \beta, I}$. The choices made enable the locality of the slip check in terms of already defined variables. Moreover, since only the components of quantities with respect to the curvilinear coordinate system are being employed, the overall formulation ensures objectivity automatically (cf. [22,23]).

This completes the treatment of tangential contact apart from linearization, which is treated in Section 3.

\subsection{Alternative recovery procedures}

\subsubsection{Lumped recovery and consistency statement}

In order to avoid the computation of the inverse ${ }^{2} \hat{\Psi}^{I J}$ and the matrix operations resulting from its use, it is advantageous to pursue an approximate treatment. For this purpose, $\widehat{\Psi}^{I I}$ is replaced by $\delta_{I J} / \psi^{I}$ where $\delta_{I J}$ is the Kronecker delta. For instance, (2.28) may now be stated using (2.30) as

$\tau_{\alpha}^{I}=\frac{\bar{\tau}_{\alpha}^{I, n}}{\psi^{I}}+\epsilon_{T}\left(\sum_{J} \frac{\bar{a}_{\alpha \beta}^{I J}}{\psi^{I}} \gamma_{T}^{\beta, J}-\frac{\bar{\Lambda}^{I} S_{\alpha}^{I}}{\psi^{I}}\right)$.

In [1], a consistency statement was derived for such an approximation in the context of an augmented Lagrange multiplier setting with Uzawa iterations. In the frictional formulation, this requirement arises already without augmentations due to the history variable $\tau_{\alpha}^{I, n}$. Indeed, the update (2.31) applied at vanishing incremental updates implies that defining

$\bar{\tau}_{\alpha}^{I, n}=\tau_{\alpha}^{I, n} \psi^{I}$

is necessary since the simplified recovery in (2.31) is not compatible with the original projection $\bar{\tau}_{\alpha}^{I, n}=\left\langle N^{I} \tau_{\alpha}^{n}\right\rangle$ that is intrinsic in (2.20). Eq. (2.31) may then be stated as

$\tau_{\alpha}^{I}=\tau_{\alpha}^{I, n}+\epsilon_{T}\left(\sum_{J} \frac{\bar{a}_{\alpha \beta}^{I J}}{\psi^{I}} \gamma_{T}^{\beta, J}-\Lambda^{I} s_{\alpha}^{I}\right)$,

where $\Lambda^{I}:=\bar{\Lambda}^{I} / \psi^{I}$. This result formed the basis of the studies in [35] without derivation. ${ }^{3}$

\subsubsection{Formulation in terms of mixed variables for lumped recovery}

The consistency condition for the normal contact treatment with a lumped recovery ensures the satisfaction of the HertzSignorini-Moreau conditions by the mixed mortar quantities as well provided $\psi^{I}>0$, which is ensured in this study due to the use of NURBS or linear Lagrange basis functions. Presently, the consistency condition (2.32) also induces the convenient fact that the slip surface is not violated by the mixed quantities either, provided the same norm (2.22) is employed. This is easily seen since the discrete slip criterion is

$\bar{\sigma}^{I}\left(\bar{\tau}^{I}, \bar{p}_{N}^{I}\right)=\left\|\bar{\tau}^{I}\right\|-\mu \bar{p}_{N}^{I}=\psi \psi^{I}\left\|\tau^{I}\right\|-\mu \bar{p}_{N}^{I} \leqslant 0$

and therefore the criterion in terms of mixed quantities is

$\sigma^{I}\left(\tau^{I}, p_{N}^{I}\right):=\left\|\tau^{I}\right\|-\mu p_{N}^{I} \leqslant 0$

such that $\sigma^{I, t r}=\sigma^{I}\left(\tau^{I, t r}, p_{N}^{I}\right)>0$ indicates slip. Moreover, noting

\footnotetext{
${ }^{2}$ Numerically, one does not necessarily need to explicitly determine the inverse although, due to multiple uses, this is advantageous in the present case.

3 There is an error in Eq. (2.16) of [35]. The tangential kinematic variable should be the projected one.
}

$m_{\alpha \beta}^{I} \frac{\partial \sigma^{I}}{\partial \tau_{\beta}^{I}}=\frac{\tau_{\alpha}^{I, t r}}{\left\|\tau^{I, t r}\right\|}=s_{\alpha}^{I}$,

when the slip criterion (2.35) is violated $\tau_{\alpha}^{I}=\mu p_{N}^{I} s_{\alpha}^{I}$ holds.

In Eq. (2.33), an element-level connectivity arising from the trial update tractions and the locality of the stick/slip check are clearly observed. The lumped recovery approach allows further simplification and matching a formulation that was presented in [36]. The row-sum lumping of the projected metric $\bar{a}_{\alpha \beta}^{I J}$ and its subsequent scaling leads to the definitions

$\bar{a}_{\alpha \beta}^{I}:=\left\langle N^{I} a_{\alpha \beta}\right\rangle, \quad m_{\alpha \beta}^{I}:=\frac{\bar{a}_{\alpha \beta}^{I}}{\psi^{I}}$.

The final update then reduces (2.33) to an entirely local relation between the mixed quantities:

$\tau_{\alpha}^{I}=\tau_{\alpha}^{I, n}+\epsilon_{T}\left(m_{\alpha \beta}^{I} \gamma_{T}^{\beta, I}-\Lambda^{I} s_{\alpha}^{I}\right)$.

This modification, which eliminates the element-level connectivity and requires retaining only the metric $m_{\alpha \beta}^{I}$ in the formulation, considerably simplifies the implementation and even appears necessary for consistency within the lumped recovery approach. Nevertheless, for the numerical investigations carried out, it was observed that the results remain unaffected with respect to (2.33).

\subsubsection{Unconstrained recovery}

The lumped approach is more appropriately viewed as an approximation of an unconstrained recovery procedure for the mixed tangential variables that is also a generalization of the constrained approach. In this case,

$\gamma_{T}^{\alpha, I}=\sum_{J} \widehat{\Phi}^{I J} \chi^{J} \bar{g}_{T}^{\alpha . J}$

replaces (2.15) where $\widehat{\Phi}^{I J}$ is associated with the inverse of $\Phi^{I J}$. Moreover, the history variable distribution $\tau_{\alpha}^{n}$ should be updated to account for changes in the active set in order to ensure consistency among the mixed and projected quantities associated with the augmented traction distribution $\tau_{\alpha}$. This is achieved by computing the projected quantities $\bar{\tau}_{\alpha}^{I, n}$ in a first step and subsequently updating the mixed quantities $\tau_{\alpha}^{I, n}$ using

$\tau_{\alpha}^{I, n}=\sum_{J} \widehat{\Phi}^{I J} \chi^{J} \bar{\tau}_{\alpha}^{J, n}$

which retains the original values for $\tau_{\alpha}^{I, n}$ unless there is a change in the active set.

This unconstrained recovery procedure ensures smooth evolutions of $\gamma_{T}^{\alpha, I}$ for smooth evolutions of $\bar{g}_{T}^{\alpha, I}$ both for large changes in the active set as well as in the transition from stick to slip, thereby avoiding jumps in the contact interactions that may be observed with the constrained formulation at coarse discretizations. However, the computational cost for the unconstrained formulation is higher since now all slave elements contribute to the evaluation of the contact integrals at all times. The differences between the constrained and unconstrained formulations trivially vanish when all nodes are active. In all the examples to be provided in Section 4, the same recovery procedure is employed for the normal and tangential parts of the contact treatment.

\subsection{Uzawa augmentation}

\subsubsection{Iterative scheme}

The implementation of an augmented Lagrange multiplier approach in the context of Uzawa iterations [3] now easily follows for all recovery procedures. With the default constrained recovery procedure, the augmentation of the pressure is complemented by the replacement $\bar{\tau}_{\alpha}^{I, n} \leftarrow \bar{\tau}_{\alpha}^{I,(k)}$ in Eq. (2.28), which updates the 
traction at the $k$ th Uzawa augmentation of time step $n+1$ with $\bar{\tau}_{\alpha}^{I,(0)}=\bar{\tau}_{\alpha}^{I, n}$. With the approximate lumped recovery, the consistency statement $\bar{\tau}_{\alpha}^{I,(k)}=\tau_{\alpha}^{I,(k)} \psi^{I}$ defines the projected quantities instead of the original definition. Alternatively, $\tau_{\alpha}^{I, n}$ may be replaced by $\tau_{\alpha}^{I,(k)}$ in (2.33) or (2.38) to avoid defining projected tractions. Finally, with the unconstrained recovery one proceeds as for the constrained case except that $\tau_{\alpha}^{I,(k)}$ must be updated as in (2.40). In all cases, unindexed quantities in the equations are now understood to belong to augmentation step $k+1$. The augmentations are continued until an iterative error measure $\mathfrak{f}^{(k+1)}$ satisfies a convergence criterion to within a given tolerance ToL.

\subsubsection{A comparison}

It is important to point out that the augmented Lagrangian approach without Uzawa iterations, which forms a basis for the related semi-smooth Newton schemes mentioned earlier in the introduction, was originally presented in [29] also as a mixed approach. Here, the terminology mixed referred to the combination of penalty and duality methods such that the advantages of both were incorporated into a single framework. The present use of the terminology, on the other hand, follows the usage in [2] which took as its basis the classical Veubeke-Hu-Washizu variational principle [37] and its application in the numerical analysis of incompressible materials [38]. The former usage refers to the enforcement of the contact constraints whereas the present usage refers to their discretization. ${ }^{4}$ In particular, the appearance of the history of the tangential traction in the classical formulation (2.7) that forms the basis of the novel formulation proposed in (2.9) should not be confused with an augmented Lagrangian framework. Indeed, for both normal and tangential contact, the functionals stated are strictly for the penalty setting, possibly followed by Uzawa iterations as summarized. This is clear for normal contact due to the absence of a Lagrange multiplier in the formulation. The history variable may likewise be eliminated from (2.8) and (2.9) by defining an elastic tangential gap [4] to obtain an approach that is closer to the normal part, although this choice was not pursued in this work. A mixed framework that is based on the original augmented Lagrangian scheme for constraint enforcement and which would be prone to a Newton-Raphson type solution approach would require a different functional as a starting point.

\section{Linearization of the mixed formulation}

While constrained recovery is not the most satisfactory among the three approaches presented (see Section 4.5), it naturally bridges the remaining two. For this reason, the algorithm for the treatment of tangential contact with a constrained recovery is summarized in Table 1.

Within a Newton-Raphson approach, $-\Delta \delta \mathcal{G}^{c}$ contributes to the tangent matrix:

$-\Delta \delta \mathcal{G}^{c}=-\Delta \delta \mathcal{G}_{N}^{c}-\Delta \delta \mathcal{G}_{T}^{c}$.

Concentrating on the tangential contribution, it can be expressed as

$-\Delta \delta \mathcal{G}_{T}^{c}=\int_{\partial \mathcal{R}_{0}^{c}}\left(\Delta \delta g_{T}^{\alpha} \tau_{\alpha}+\delta g_{T}^{\alpha} \Delta \tau_{\alpha}\right) d A$.

Here, $\Delta \delta g_{T}^{\alpha}=\Delta \delta \xi^{\alpha}$ is purely kinematic in nature and hence the treatment of the first term which yields a symmetric form is standard-see $[3,4]$. To proceed with the second term, which is nonsymmetric in general in the regularized continuum formulation as well [3, p. 161], in view of (2.12) and (2.19)

\footnotetext{
${ }^{4}$ The use of the terminology arises in other contexts as well, e.g. [39].
}

$\Delta \tau_{\alpha}=\sum_{I} N^{I} \chi^{I} \Delta \tau_{\alpha}^{I}, \quad \Delta \tau_{\alpha}^{I}=\sum_{J} \widehat{\Psi}^{I J} \chi^{J} \Delta \bar{\tau}_{\alpha}^{I}$

so that

$\Delta \tau_{\alpha}=\sum_{I} \sum_{J} N^{I} \chi^{I} \widehat{\Psi}^{I J} \chi^{J} \Delta \bar{\tau}_{\alpha}^{J}$

where the indicators are explicitly denoted to highlight vanishing terms. Therefore,

$\int_{\partial \mathcal{R}_{0}^{c}} \delta g_{T}^{\alpha} \Delta \tau_{\alpha} d A=\sum_{I} \sum_{J} \delta \bar{g}_{T}^{\alpha, I} \chi^{I} \widehat{\Psi}^{I J} \chi^{J} \Delta \bar{\tau}_{\alpha}^{J}$,

where

$\delta \bar{g}_{T}^{\alpha, I}=\left\langle N^{I} \delta g_{T}^{\alpha}\right\rangle$

has been made use of. The term $\delta g_{T}^{\alpha}$ is standard. The linearization $\Delta \bar{\tau}_{\alpha}^{J}$ depends on the stick/slip status of the node. These are treated separately to highlight the main steps of linearization. While the contributions to the residual vector associated with the variations $\left\{\delta x_{i}^{A}, \delta y_{i}^{A}\right\}$ follow from standard treatments, explicitly identifying the contributions to the tangent matrix entries $K_{i j}^{A B}$ which relate the variations $\left\{\delta x_{i}^{A}, \delta y_{i}^{A}\right\}$ to the increments $\left\{\Delta x_{j}^{B}, \Delta y_{j}^{B}\right\}$ requires some additional algebraic effort but is straightforward. In order to employ the lumped treatment of Section 2.4.1, $\widehat{\Psi}^{I I}$ is replaced by $\delta_{I J} / \psi^{I}$. For the unconstrained approach of Section 2.4.3, $\widehat{\Psi}^{I J}$ is replaced everywhere by $\widehat{\Phi}^{I J}$ and $\chi^{I}$ is removed from (3.5) as well as from all terms emanating from it.

It is remarked that although the stick formulation is motivated by the potential (2.7), the variation of the metric was omitted from the weak form in order to simplify the algorithmic implementation and to subsequently construct the mixed formulation as an extension of the approach pursued in [3]. Hence, one can trace the lack of stick symmetry to this simplification. It can easily be shown that the incorporation of the missing variation $\delta a_{\alpha \beta}$ in the weak form would restore the symmetry for the stick state. A qualitatively similar issue arises whenever the weak form is taken as the starting point for the penalty regularization of normal contact on the deformed configuration. A non-symmetric tangent arises unless the variation of the differential area, which would naturally emanate from a contact potential, is explicitly incorporated into the weak form [28]. Presently, the absence of $\delta a_{\alpha \beta}$ complies with the classical update for $\tau_{\alpha}$ in the stick state that is induced by a penalty regularization starting from the weak form. Therefore, one concludes that the classical update does not comply with the exact variation of the potential formulation. An update that would induce a symmetric contribution in the stick state was proposed in [40] based on an improved geometric interpretation of the tangential contact interactions. In this work, the analogy to the classical update will be retained.

\subsection{Stick state}

In the stick state, using (2.17) and (2.20),

$$
\Delta \bar{\tau}_{\alpha}^{J}=\Delta \bar{p}_{T \alpha}^{J}=\epsilon_{T} \sum_{K}\left(\Delta \bar{a}_{\alpha \beta}^{J K} \gamma_{T}^{\beta, K}+\bar{a}_{\alpha \beta}^{J K} \Delta \gamma_{T}^{\beta, K}\right),
$$

where, via (2.18),

$\Delta \bar{a}_{\alpha \beta}^{J}=\left\langle N^{J} \Delta a_{\alpha \beta} N^{K}\right\rangle$,

with $\Delta a_{\alpha \beta}$ as a standard term and, via (2.15),

$\Delta \gamma_{T}^{\alpha, K}=\sum_{L} \widehat{\Psi}^{K L} \chi^{L} \Delta \bar{g}_{T}^{\alpha, L}$,

which can be evaluated by making use of (3.6). 
Table 1

Algorithm for tangential contact treatment with Uzawa augmentation and constrained recovery.

(Normal contact contributions are available from [1]. Contact active set $\mathcal{A}$ is accordingly updated.)

1. Initialize. The closest-point projection $\xi^{\alpha, n}$ and the mixed kinetic variable $\tau_{\alpha}^{l, n}=\tau_{\alpha}^{l,(0)}$ at time/load step $n$ are known. Within a Newton-Raphson iteration of the $k$ th

Uzawa augmentation at time/load step $n+1$, compute the incremental kinematic variable and the augmentation tractions:

$$
g_{T}^{\alpha}=\xi^{\alpha}-\xi^{\alpha, n}, \quad \tau_{\alpha}^{(k)}=\sum_{I} N^{I} \tau_{\alpha}^{I,(k)}
$$

2. Projected mortar variables. Compute projections of kinematic and kinetic variables for $I \in \mathcal{A}$ :

$$
\bar{g}_{T}^{\alpha, I}=\left\langle N^{I} g_{T}^{\alpha}\right\rangle, \quad \bar{\tau}_{\alpha}^{I,(k)}=\left\langle N^{I} \tau_{\alpha}^{(k)}\right\rangle
$$

3. Mixed kinematic variable recovery. Compute the nodal values of the mixed kinematic variable:

$$
\gamma_{T}^{\alpha, I}=\sum_{J} \widehat{\Psi}^{I J} \chi^{J} \bar{g}_{T}^{\alpha J}
$$

4. Projected trial tractions. Update projected kinetic variable assuming stick:

$$
\bar{p}_{T \alpha}^{I, t r}=\epsilon_{T} \sum_{J} \bar{a}_{\alpha \beta}^{I J} \gamma_{T}^{\beta, J} \rightarrow \bar{\tau}_{\alpha}^{I, t r}=\bar{\tau}_{\alpha}^{I,(k)}+\bar{p}_{T \alpha}^{I, t r}
$$

5. Slip surface evaluation. Check locally for slip using the projected variables:

$$
\left\|\bar{\tau}^{I, t r}\right\|^{2}=\bar{\tau}_{\alpha}^{I, t r} m^{\alpha \beta, I} \bar{\tau}_{\beta}^{I, t r} \rightarrow \bar{\sigma}^{I, t r}=\left\|\bar{\tau}^{I, t r}\right\|-\mu \bar{p}_{N}^{I}:\left\{\begin{array}{l}
\text { if } \leqslant 0 \text { then Stick }: \bar{\tau}_{\alpha}^{I}=\bar{\tau}_{\alpha}^{I, t r} \\
\text { if }>0 \text { then Slip }: \bar{\tau}_{\alpha}^{I}=\mu \bar{p}_{N}^{I} s_{\alpha}^{I}
\end{array}\right.
$$

6. Mixed kinetic variable recovery. Compute the nodal values of the mixed tractions:

$$
\tau_{\alpha}^{I}=\sum_{J} \widehat{\Psi}^{I J} \bar{\tau}_{\alpha}^{J}
$$

7. Local traction. Compute the updated local traction for the weak form evaluation and solution:

$$
\tau_{\alpha}=\sum_{I} N^{I} \tau_{\alpha}^{I} \rightarrow \delta \mathcal{G}_{T}^{c}=-\int_{\partial \mathcal{R}_{o}^{c}} \delta \xi^{\alpha} \tau_{\alpha} d A
$$

8. Check convergence. Estimate the error in augmentation iteration $k+1$ and reiterate unless $\mathfrak{E}^{(k+1)}<$ Tol

\subsection{Slip state}

If the slip criterion (2.23) indicates slip, $\Delta \bar{\tau}_{\alpha}^{J}$ from (3.7) corresponds to the trial quantity $\Delta \bar{\tau}_{\alpha}^{J, t r}$. Now, the linearization of $(2.26)$ delivers

$\Delta \bar{\tau}_{\alpha}^{J}=\mu\left(\Delta \bar{p}_{N}^{J} s_{\alpha}^{J}+\bar{p}_{N}^{J} \Delta s_{\alpha}^{J}\right)$.

The first linearization has been treated in normal contact. Using (2.27) and standard calculations [3,4], the second linearization reads

$\Delta s_{\alpha}^{J}=\frac{\Delta \bar{\tau}_{\beta}^{J, t r}}{\left\|\bar{\tau}^{J}, t r\right\|}\left(\delta_{\beta}^{\alpha}-s_{\alpha}^{J} m^{\beta \gamma, J} S_{\gamma}^{J}\right)-\frac{1}{2} s_{\alpha}^{J} S_{\beta}^{J} S_{\gamma}^{J} \Delta m^{\beta \gamma, J}$.

The linearization in the first term has been treated within the stick formulation. Recalling the definition (2.25), the linearization $\Delta m^{\beta \gamma, I}=\Phi^{I I} \Delta \bar{a}^{\beta \gamma, I I}$ in the second term is most easily carried out by noting

$\Delta \bar{a}^{\beta \gamma, I I}=-\bar{a}^{\beta \theta, I I} \Delta \bar{a}_{\theta \delta}^{I I} \bar{a}^{\delta \gamma, I I}$,

where $\Delta \bar{a}_{\theta \delta}^{I I}$ has been treated in (3.8).

Although not pursued in this work, it is remarked that in the context of Uzawa augmentations algorithmic symmetrization [3] by keeping $\bar{p}_{N}^{I}$ fixed for the frictional part throughout an augmentation step could possibly be used to considerably facilitate the linearization and hence the ensuing numerical implementation.

\section{Numerical investigations}

In this section, major aspects of the developed frictional mortarbased contact formulation are investigated within a quasistatic setting. In Section 4.1, the local solution quality is investigated by monitoring the traction distributions for the classical HertzMindlin contact problem with two deformable bodies. Patch tests with tied interfaces are conducted in two variants of the classical flat interface problem, in Section 4.2 with a wedge-like interface and in Section 4.3 for a curved interface. The global solution quality is assessed in Section 4.4, where a tire is dragged on a deformable foundation at large compressive loads, by monitoring the contact forces. Finally, additional global solution comparisons of different types of mixed formulation are carried out in Section 4.5 through the rotating contact of two elastic bodies.

While constrained and lumped recovery approaches delivered very close results for the frictionless setup both locally and globally in many instances even at coarse resolutions, this is not the case for frictional contact. On the other hand, while constrained and unconstrained recovery results differ even for such cases, presently the example of Section 4.5 is the only case where unconstrained recovery is needed to provide performance improvement. For this reason, it is not considered in earlier sections and emphasis is placed on the former two recovery approaches. In all examples, the volume is discretized with either linear Lagrange or quadratic NURBS elements, the latter based on the recent developments in $[41,42,36,43,35,44]$.

The notation, the choices for the numerical discretization as well as the constitutive models and parameters follow [1] and will not be repeated.

\subsection{Classical Hertz-Mindlin contact}

The classical Hertz-Mindlin contact problem is considered within a plane-strain setting with two deformable bodies. The classical problem geometry with two cylindrical bodies was provided 
in [1] and hence is only briefly described. Under frictional contact with $\mu=0.25$, the upper body is first displaced downwards in ten steps through $\Delta_{N}=0.005$ followed by a tangential displacement of $\Delta_{T}=0.001$ through ten steps. See also $[23,28]$ for alternative setups. $\epsilon_{N}=10, \epsilon_{T}=1$ and $\mathrm{ToL}=0.001$ are chosen together with non-matching discretizations. To ensure an accurate evaluation of the contact integrals, 30 integration points are employed. This high number minimizes any possible influence of the integration error due to the lack of an explicit intermediate integration surface, although the results were verified to be virtually the same when 10 points were used as in the following sections.

Results of the analysis are presented in Fig. 1. Among other alternatives, the approximate analytical solution chosen for this problem assumes that the pressure distribution is unaffected by the tangential tractions [45]. It is observed that, due to more extensive coupling among the mixed kinematic variables, constrained recovery results are more oscillatory. However, the magnitude of the oscillations decreases considerably with mesh refinement, indicating local convergence. At the fine resolution, there is a good agreement with the analytical solution for both recovery methods. It is additionally observed that, since the tangential constraints are enforced through the projected mortar quantities, the slip criterion can be violated in terms of the mixed kinematic quantities for constrained recovery. On the other hand, in agreement with the analysis Section 2.4.2, it is observed that lumped recovery guarantees that the constraints are satisfied in terms of mixed quantities as well. It is remarked that since $a_{11}$ is a constant throughout the contact zone, a convenient traction scaling has been employed in Fig. 1 to demonstrate this fact. In general, the slip criterion should strictly be checked via (2.35). Finally, the effect of switching the master/slave designations is demonstrated in Fig. 2. Since the original master resolution in the tangential direction is half the slave one, the influence is considerable at coarse resolutions and stronger than the influence on the pressure distribution. It is remarked that only eight nodes are active throughout the whole contact interface for the coarse
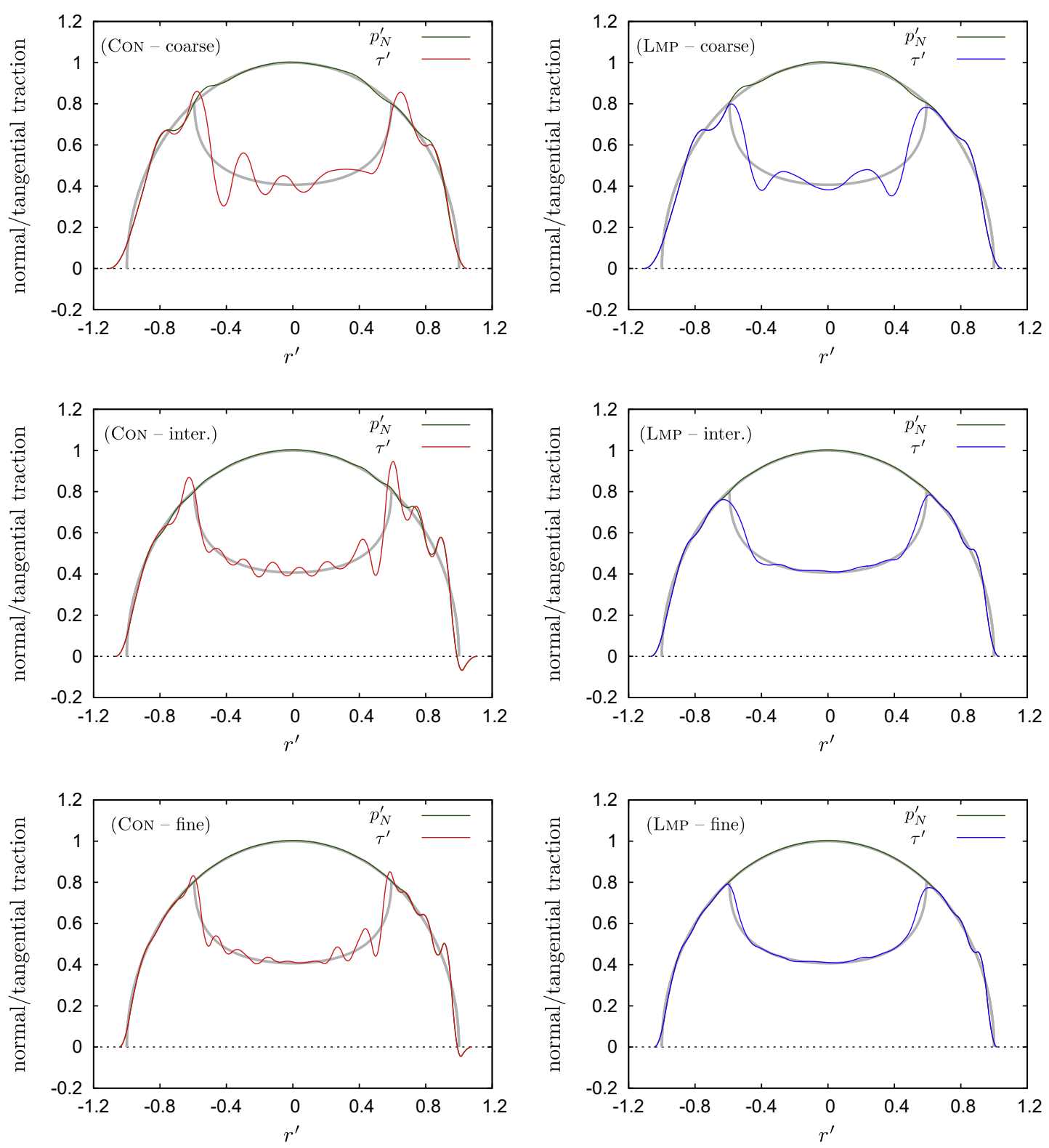

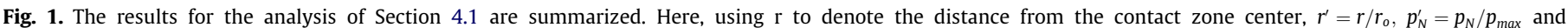

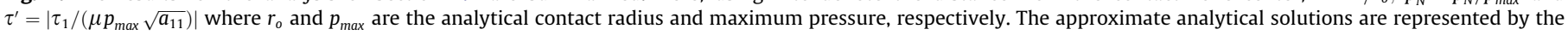
gray lines. 

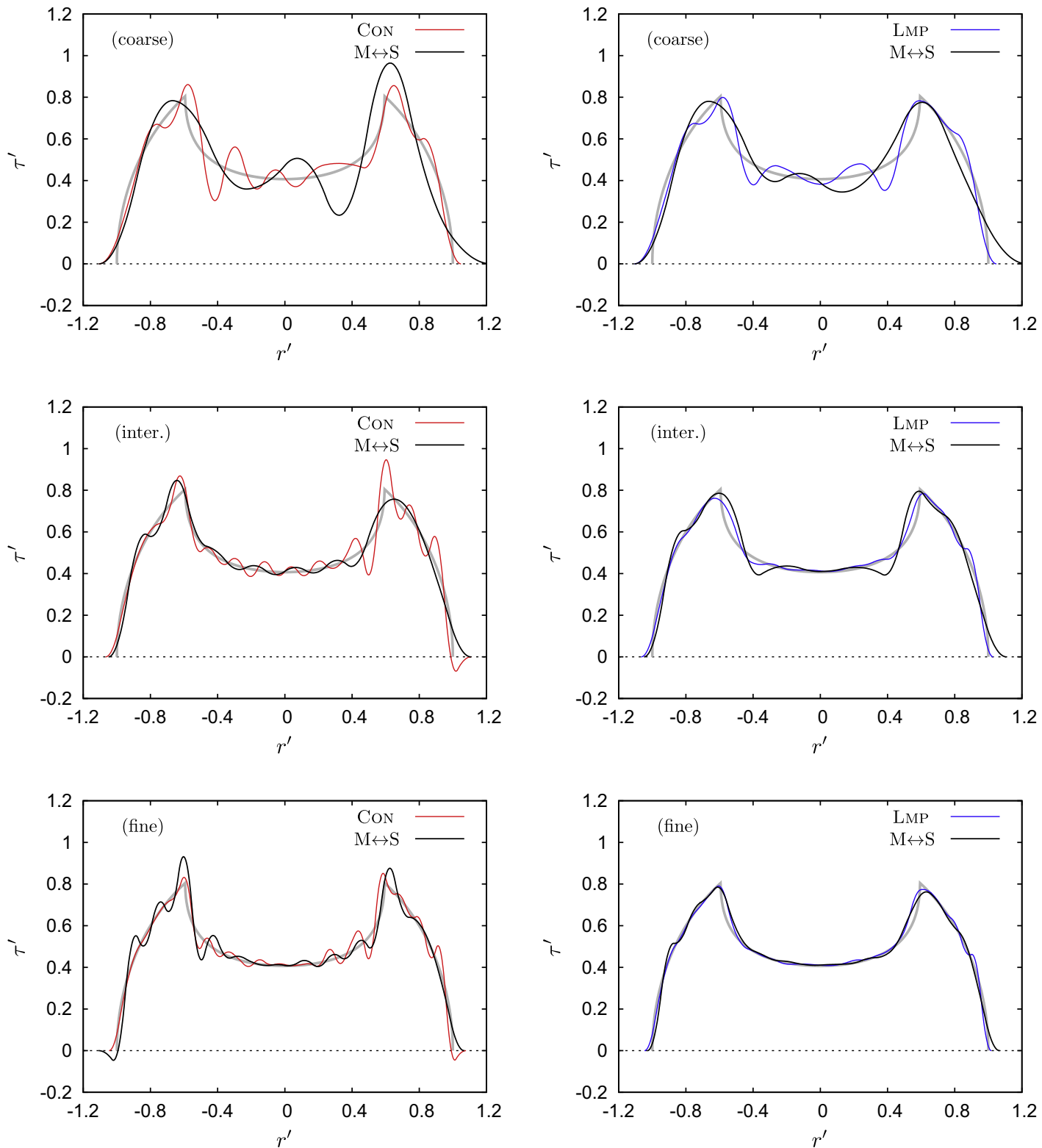

Fig. 2. The analysis of Fig. 4.1 is repeated. By default, the upper body is the slave. $M \leftrightarrow S$ indicates switching master and slave choices.

resolution. However, this sensitivity is already small for the fine resolution.

\subsection{Patch test I: inclined interface}

The classical patch test setup assumes frictionless contact conditions at the flat interface between two bodies. Presently, this setup is modified by deforming the interface to a wedge-like form and the upper body is again displaced vertically. Here, and in the next section, only the constrained recovery approach is considered since for a tied interface this approach is equivalent to the unconstrained one and also delivers an identical performance with lumped recovery. In order to ensure that the bodies will deform as one, tangential constraints must be activated. Here, the surfaces are initially slightly penetrating each other. Normal and tangential contact constraints are activated for the whole interface while stick is enforced, furnishing a domain decomposition algorithm that is suitable for Lagrange or NURBS basis functions. See also [46] for a domain decomposition approach where NURBS basis functions are employed. To ensure high accuracy, $\epsilon_{N}=\epsilon_{T}=10^{6}$ and
ToL $=10^{-9}$ are chosen. Lagrange elements are used with ten integration points per interface direction to evaluate the contact integrals.

The problem geometry and results are summarized in Fig. 3. While the bodies deform approximately as one at this high compression ratio, there are slight oscillations in the stress. However, these oscillations are not due to approximate integration but rather due to the discretization nature for the contact interactions. This is clearly observed in Fig. 4. In the exact solution of this problem, the pressure is a constant but the tangential traction distributions display a jump along the inner interface corners. Since this jump is not captured through the interpolation of the mixed quantities, an approximate traction distribution is obtained for the chosen discretization, the quality of which improves with mesh refinement.

\subsection{Patch test II: curved interface}

When tied interfaces are curved, the patch test additionally displays surface locking associated with an over-constrained contact 


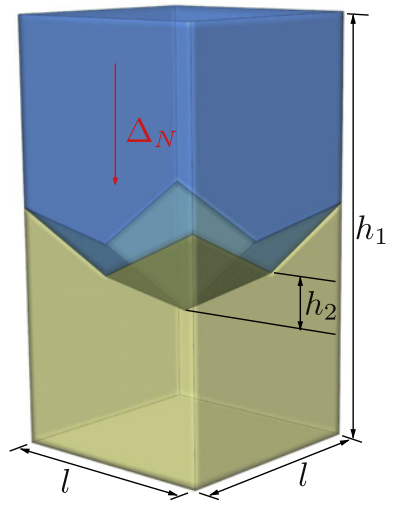

(a) problem geometry

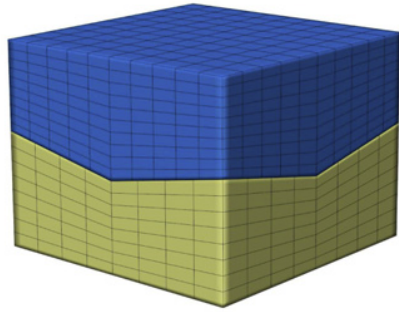

(b) deformed configuration

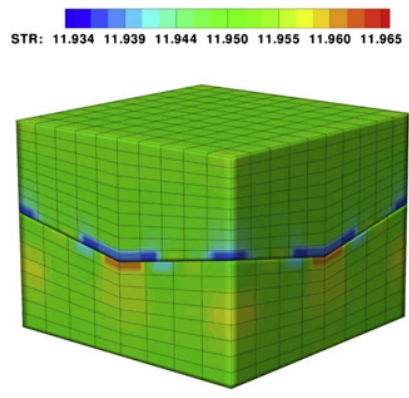

(c) stress distribution

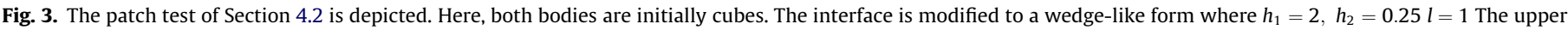

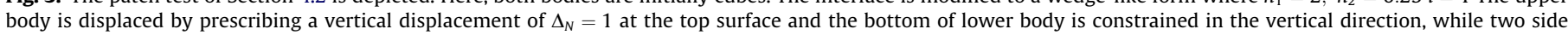

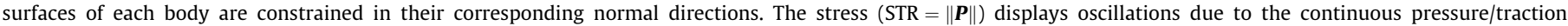
discretization.
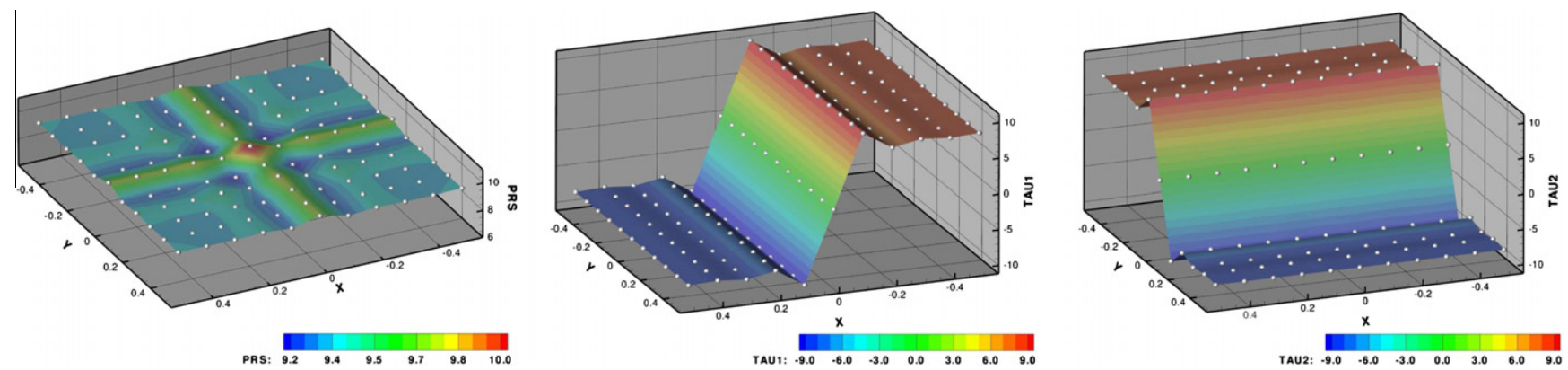

Fig. 4. For the patch test of Fig. 3 , the pressure $\left(\mathrm{PRS}=p_{N}\right)$ and tangential traction (TAU1 $=\tau_{1}$, TAU2 $\left.=\tau_{2}\right)$ distributions are displayed.

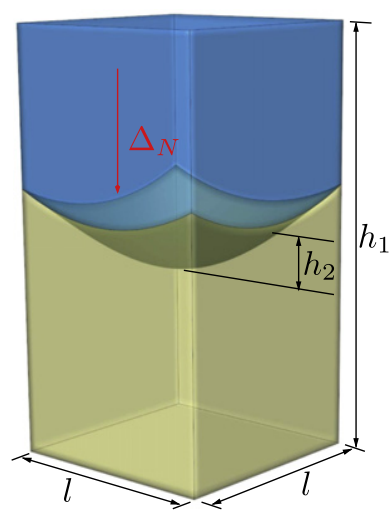

(a) problem geometry

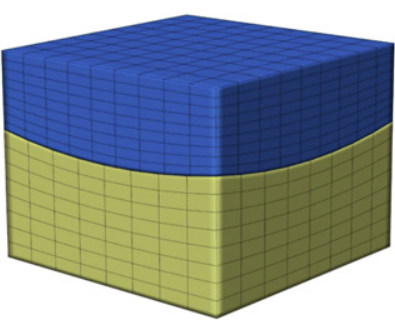

(b) constrained recovery

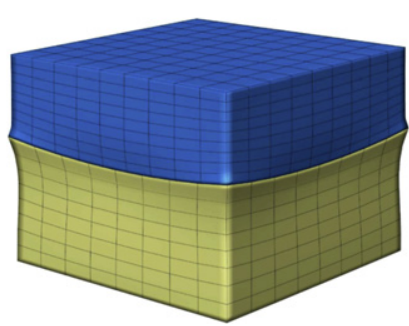

(c) a locking formulation

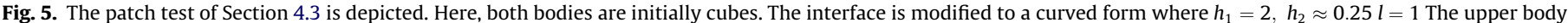

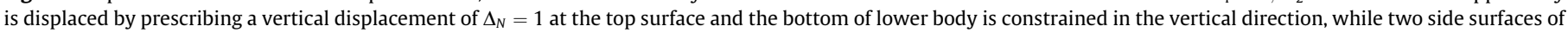
each body are constrained in their corresponding normal directions. The locking contact algorithm enforces the constraints at the integration points.

formulation [8-10]. For this purpose, the geometry and discretization of Fig. 5 are considered. As in Section 4.2, $\epsilon_{N}=\epsilon_{T}=10^{6}$ and $\mathrm{ToL}=10^{-9}$ are chosen to ensure high accuracy, together with Lagrange elements and ten integration points along each interface direction.

All recovery approaches allow the bodies to deform as one-see Fig. 5. For comparison, the constraints are enforced at the integration points of the interface elements based on the continuum approach of Section 2.1. This formulation is over-constrained and hence only a single solve with the chosen penalty parameters is carried out. For such a method Uzawa iterations will also lock, in the sense that the results degrade with each iteration and convergence is not attained for very small tolerances [9]. For all the examples in this work, ToL $=0.001$ is already a very stringent requirement that cannot be attained by the integration point formulation. The non-physical deformation of the interface is also 
clearly observed. The classical node-to-segment/surface algorithm also does not pass this patch test, although it is not over-constrained, since the forces are not transmitted correctly [10]. It is remarked that the mortar constraints are not satisfied in the reference configuration of this patch test without special modifications of the mesh [21] or the constraints [10]. The implication of this observation is that stresses are induced in the vicinity of the interface even without external loading, qualitatively similar to

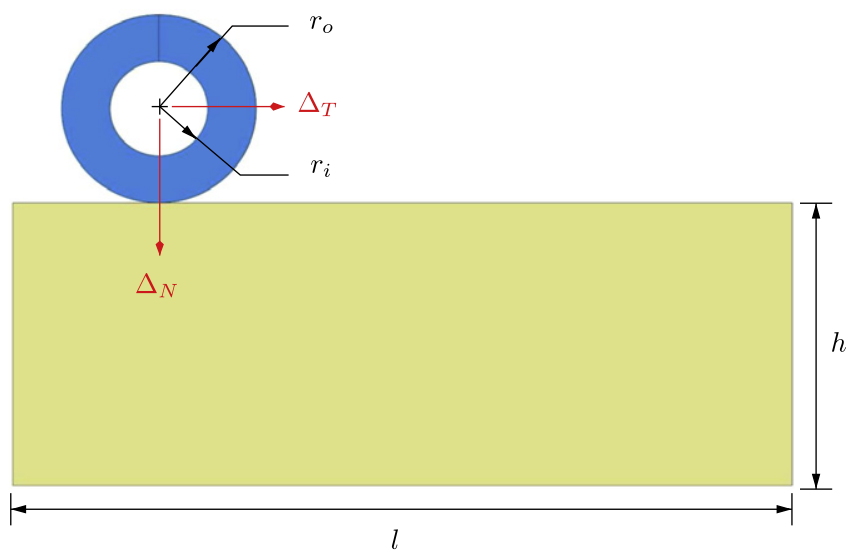

Fig. 6. The geometry of Section 4.4 is depicted. Here, $r_{o}=0.375, r_{i}=r_{o} / 2, h=$ 1.125 and $l=3$. The tire is displaced at its inner rim by first prescribing a vertical displacement of $\Delta_{N}=0.5$ followed by a tangential one with $\Delta_{T}=2$. The bottom of lower body is held fixed.

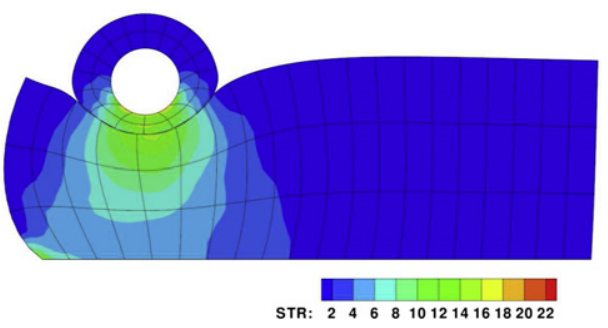

(a-1) end of compression $(\mu=0)$

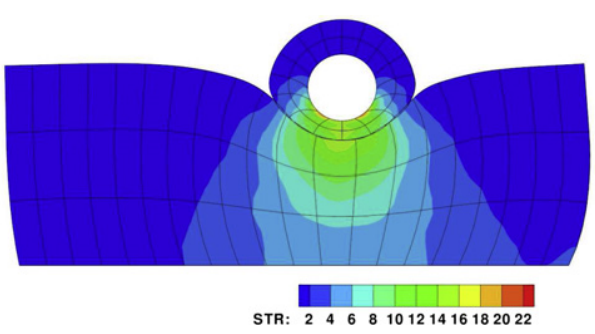

(b-1) $t=0.8(\mu=0)$

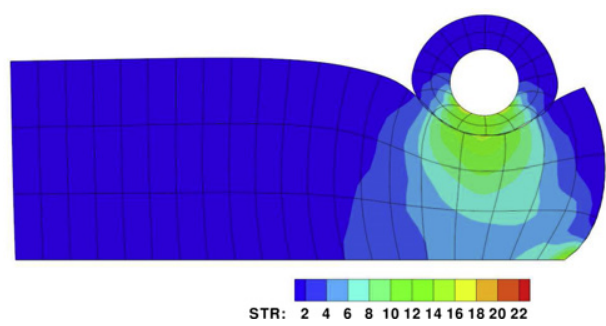

(c-1) end of dragging $(\mu=0)$
Fig. 3. An intermediate level mesh resolution was employed in this example to alleviate this effect that was not present in earlier patch test examples.

\subsection{Tire on an elastic foundation}

A tire on an elastic foundation is considered, as depicted in Fig. 6, where the tire is first pressed onto a block in 20 load steps and subsequently dragged through 20 more steps. This example is carried out in a two-dimensional setting for which the default choice for the strain energy function is replaced through its twodimensional simplification $\mathcal{W}=\frac{\Lambda_{1}}{2}(\ln J)^{2}+\frac{\Lambda_{2}}{2}\left(J^{-1} \operatorname{tr}[\boldsymbol{C}]-2\right)$. The contact variables are $\epsilon_{N}=10, \epsilon_{T}=1$, Tol $=0.001$ and $\mu=0.1$. Simulation instances are shown in Fig. 7 where the NURBS discretization employed is also shown.

Global contact interactions are monitored through the normal and tangential forces applied to the tire, as summarized in Fig. 8. Although there is large sliding at considerable compression, smooth force evolutions are obtained both for the frictional and frictionless cases. Moreover, in both cases, both recovery approaches deliver quantitatively very close results even at this coarse discretization, although the local pressure and traction distributions differ as in the Hertz-Mindlin contact example.

\subsection{Rotating contact of two elastic bodies}

As an additional example to significant contact interface evolutions, the rotating contact of two elastic bodies is considered as described in Fig. 9-see also [35]. Here, the upper body is first pressed

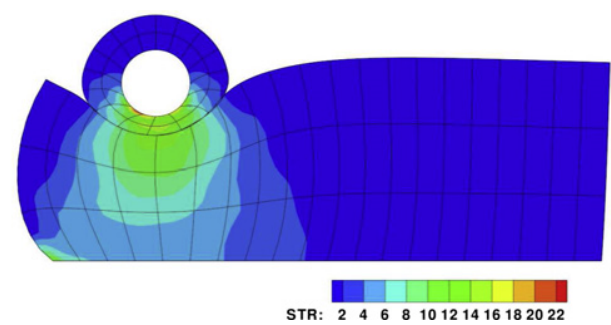

(a-2) end of compression

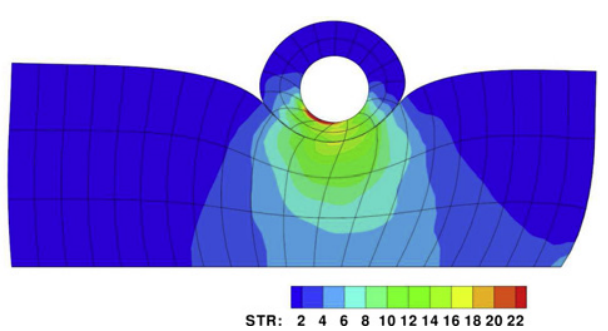

(b-2) $t=0.8$

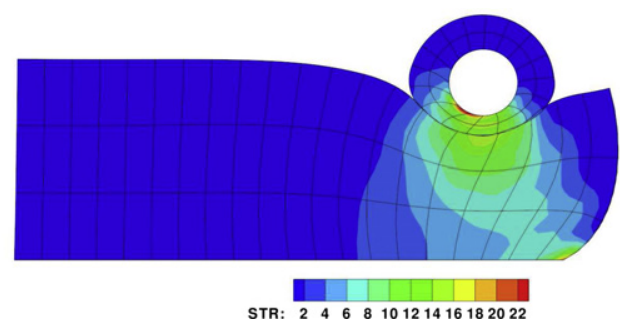

(c-2) end of dragging

Fig. 7. Simulation instances from the analysis of Fig. $6(\mathrm{STR}=\|\boldsymbol{P}\|)$ both for the frictionless and frictional cases. 

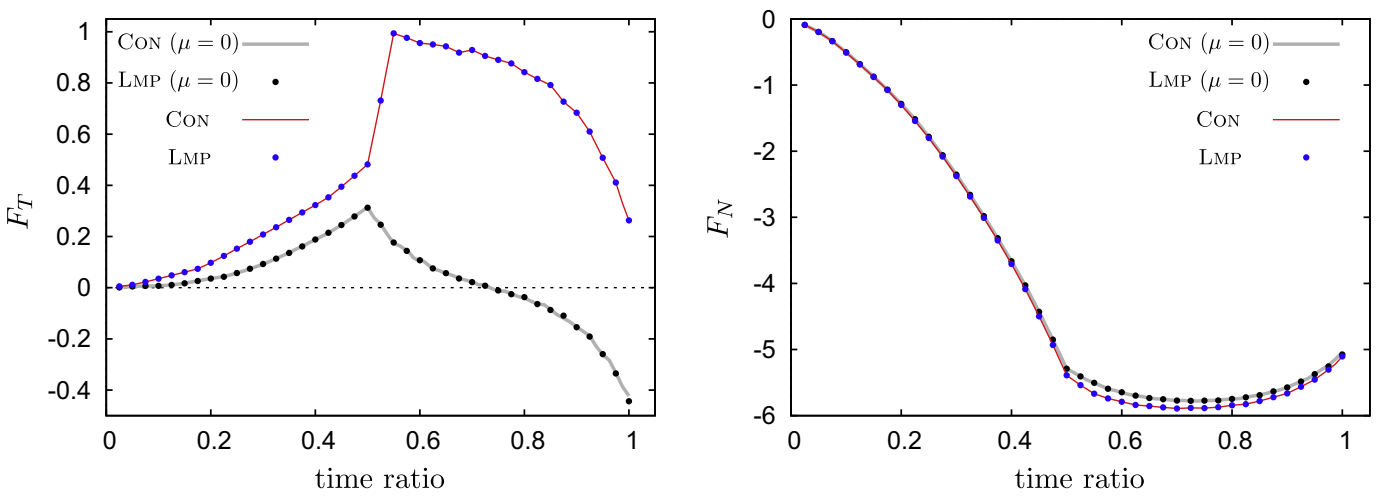

Fig. 8. The results for the analysis of Fig. 7 are summarized. Here, $F_{N}$ and $F_{T}$ are, respectively, the normal and tangential forces applied to the upper body.

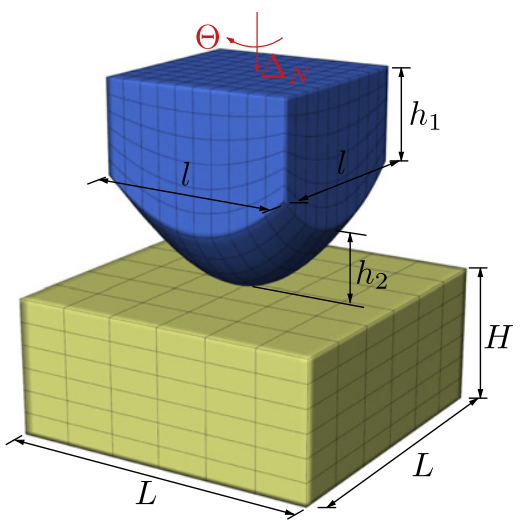

(a) problem geometry

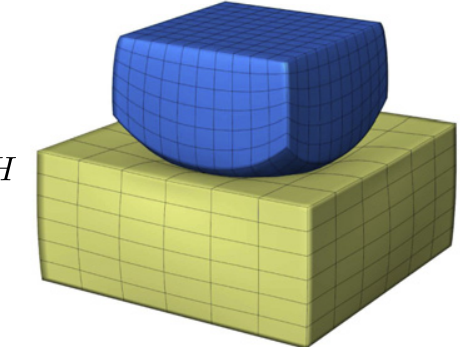

(b) end of compression

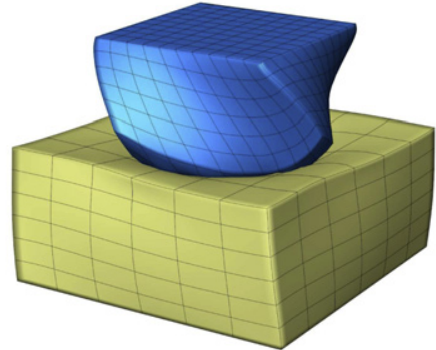

(c) end of twisting

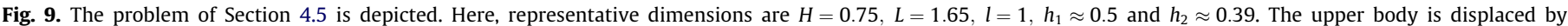

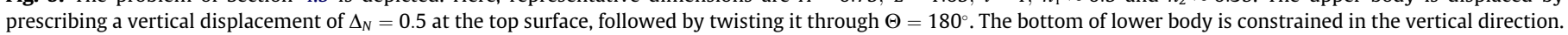
Initially, there is no gap between the surfaces.

onto the lower body in 10 load steps and subsequently rotated through 40 steps in two setups with the values 0.5 and 1 , respectively, for $\mu$. In both cases, two levels of NURBS discretization are employed. The fine resolution employs the discretization displayed in Fig. 9 while the coarse one has half as many elements in each direction. The contact variables are chosen as $\epsilon_{N}=100, \epsilon_{T}=10$ and $\mathrm{ToL}=0.01$. In this example, all recovery approaches are assessed.

The results of the simulation are summarized in Fig. 10 where the moment applied to the upper body is monitored. All recovery approaches yield quantitatively close results with the fine mesh for both friction coefficient choices. The coarse mesh results, however, highlight a problem with constrained recovery that has been described in Section 2.4.3. The evolution of the mixed tangential kinematic variables is non-smooth with respect to their projected counterparts in the transition from stick to slip. The induced jump is clearly observed, which depends on the friction coefficient. Unconstrained recovery fixes this problem. In the case of frictionless contact, a similar problem was identified [1] but therein the inconsistency was due to significant changes in the active set.
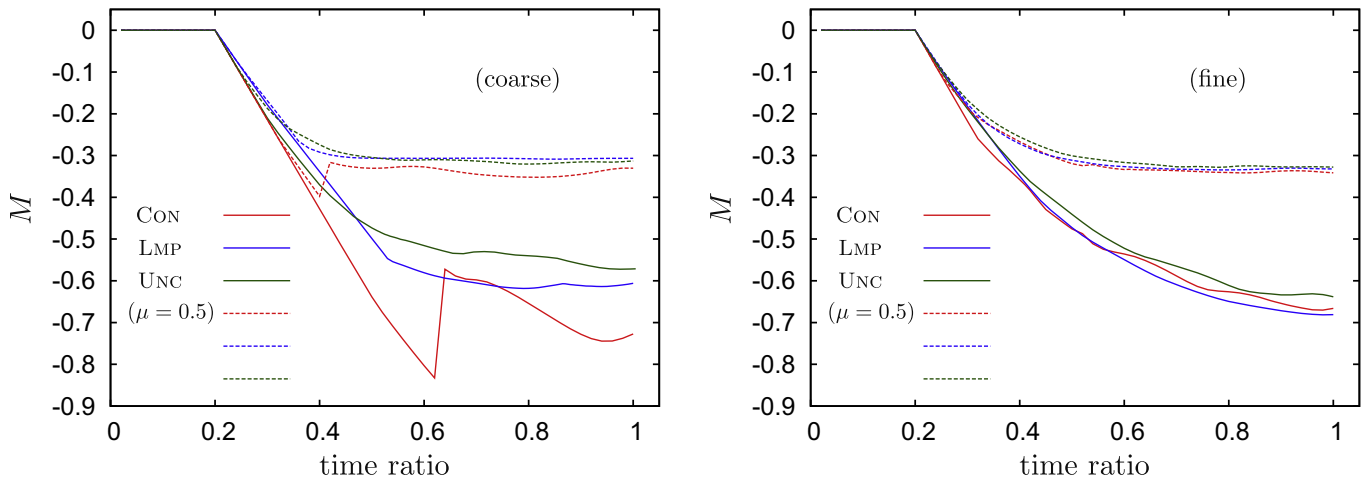

Fig. 10. The results for the analysis of Fig. 9 are summarized. Here, $M$ is the twisting moment applied to the upper body. The default value of $\mu$ is 1 . 
Presently, the active set remains almost constant throughout the twisting stage.

\section{Conclusion}

Based on the investigations for frictionless contact [1], a derivation of mortar-based treatment of frictional contact is presented. Similar to a classical proposal for the frictionless setting [2], a three-field mixed approach is constructed in terms of the tangential variables. The identification of a set of discrete mortar constraints for the tangential contact interactions is automatically delivered through the variational framework. In order to recover the corresponding mixed kinematic and kinetic variables from their projected counterparts, three closely related approaches were discussed: (i) constrained, (ii) lumped, and (iii) unconstrained. While the lumped approach appears to be computationally the most appealing, its formulation is based on the unconstrained approach that delivers the numerically less robust constrained one as a special case as well. Aspects of augmentation in the context of Uzawa iterations as well as the algorithmic linearization of the overall algorithm were presented. In order to assess the performance of the recovery approaches, two- and three-dimensional numerical investigations in the infinitesimal and finite deformation regimes were carried out. In particular, locking and patch test studies were successfully conducted together with local and global convergence monitoring.

The resulting mortar algorithm differs from earlier approaches, mainly due to the direct use of the underlying curvilinear coordinate system for the tangential treatment within mortar projections. This allows a straightforward satisfaction of the objectivity requirements. In order to extend the applicability of this algorithm to dynamic problems, it is necessary to consider the conservation of linear and angular momenta [22], which is also important in the context of domain decomposition [21] and requires the satisfaction of the mortar constraints in the reference configuration. For this purpose, the use of a local curvilinear coordinate system would allow for a reformulation of the constraints based on the approach of [10]. Finally, a three-field mixed variational formulation may also allow the derivation of approaches suitable for mortarbased thermomechanical contact analysis [32,18].

\section{Acknowledgment}

Support for this work was provided by the Scientific and Technological Research Council of Turkey (TÜBITAK) under the Career Programme Grant No. 110M661.

\section{References}

[1] İ. Temizer, A mixed formulation of mortar-based frictionless contact, Comput. Methods Appl. Mech. Engrg. 223-224 (2012) 173-185.

[2] P. Papadopoulos, R.L. Taylor, A mixed formulation for the finite element solution of contact problems, Comput. Methods Appl. Mech. Engrg. 94 (1992) 373-389.

[3] T.A. Laursen, Computational Contact and Impact Mechanics, first ed., Springer, Berlin, Heidelberg, New York, 2003 (corr. 2nd printing).

[4] P. Wriggers, Computational Contact Mechanics, second ed., Springer, Berlin, Heidelberg, New York, 2006.

[5] B. Wohlmuth, Variationally consistent discretization schemes and numerical algorithms for contact problems, Acta Numer. 20 (2011) 569-734

[6] F. Ben Belgacem, P. Hild, P. Laborde, Approximation of the unilateral contact problem by the mortar finite element method, C.R. Acad. Sci. Paris Sér. I 324 (1997) 123-127.

[7] P. Hild, Numerical implementation of two nonconforming finite element methods for unilateral contact, Comput. Methods Appl. Mech. Engrg. 184 (2000) 99-123.

[8] M.A. Crisfield, Re-visiting the contact patch test, Int. J. Numer. Methods Engrg. 48 (2000) 435-449.

[9] J.M. Solberg, R.E. Jones, P. Papadopoulos, A family of simple two-pass dual formulations for the finite element solution of contact problems, Comput. Methods Appl. Mech. Engrg. 196 (2007) 782-802.
[10] C. Hesch, P. Betsch, Transient three-dimensional domain decomposition problems: frame-indifferent mortar constraints and conserving integration, Int. J. Numer. Methods Engrg. 82 (2010) 329-358.

[11] M.A. Puso, T.A. Laursen, A mortar segment-to-segment contact method for large deformations, Comput. Methods Appl. Mech. Engrg. 193 (2004) 601-629.

[12] S. Hüeber, B.I. Wohlmuth, A primal-dual active set strategy for non-linear multibody contact problems, Comput. Methods Appl. Mech. Engrg. 194 (2005) 3147-3166.

[13] S. Hüeber, M. Mair, B.I. Wohlmuth, A priori error estimates and an inexact primal-dual active set strategy for linear and quadratic finite elements applied to multibody contact problems, Appl. Numer. Math. 54 (2005) 555-576.

[14] K.A. Fischer, P. Wriggers, Frictionless 2D contact formulations for finite deformations based on the mortar method, Comput. Mech. 36 (2005) 226244

[15] A. Popp, M.W. Gee, W.A. Wall, A finite deformation mortar contact formulation using a primal-dual active set strategy, Int. J. Numer. Methods Engrg. 79 (2009) 1354-1391.

16] C. Hesch, P. Betsch, A mortar method for energy-momentum conserving schemes in frictionless dynamic contact problems, Int. J. Numer. Methods Engrg. 77 (2009) 1468-1500.

[17] T. Cichosz, M. Bischoff, Consistent treatment of boundaries with mortar contact formulations using dual Lagrange multipliers, Comput. Methods Appl. Mech. Engrg. 200 (2011) 1317-1332.

[18] C. Hesch, P. Betsch, Energy-momentum consistent algorithms for dynamic thermomechanical problems Application to mortar domain decomposition problems, Int. J. Numer. Methods Engrg. 86 (2011) 1277-1302.

[19] G. Bayada, M. Chambat, K. Lhalouani, T. Sassi, About the mortar finite element method for non-local Coulomb law in contact problems, C.R. Acad. Sci. Paris Sér. I 325 (1997) 1323-1328.

[20] T.W. McDevitt, T.A. Laursen, A mortar-finite element formulation for frictional contact problems, Int. J. Numer. Methods Engrg. 48 (2000) 1525-1547.

[21] M.A. Puso, A 3D mortar method for solid mechanics, Int. J. Numer. Methods Engrg. 59 (2004) 315-336.

[22] M.A. Puso, T.A. Laursen, A mortar segment-to-segment frictional contact method for large deformations, Comput. Methods Appl. Mech. Engrg. 193 (2004) 4891-4913.

[23] B. Yang, T.A. Laursen, X. Meng, Two dimensional mortar contact methods for large deformation frictional sliding, Int. J. Numer. Methods Engrg. 62 (2005) 1183-1225.

[24] K.A. Fischer, P. Wriggers, Mortar based frictional contact formulation for higher order interpolations using the moving friction cone, Comput. Methods Appl. Mech. Engrg. 195 (2006) 5020-5036.

[25] M.A. Puso, T.A. Laursen, J. Solberg, A segment-to-segment mortar contact method for quadratic elements and large deformations, Comput. Methods Appl. Mech. Engrg. 197 (2008) 555-566.

[26] S. Hüeber, A. Matei, B.I. Wohlmuth, Efficient algorithms for problems with friction, SIAM J. Sci. Comput. 29 (2007) 70-92.

[27] S. Hüeber, G. Stadler, B.I. Wohlmuth, A primal-dual active set algorithm for three-dimensional contact problems with Coulomb friction, SIAM J. Sci. Comput. 30 (2008) 572-596.

[28] M. Tur, F.J. Fuenmayor, P. Wriggers, A mortar-based frictional contact formulation for large deformations using Lagrange multipliers, Comput. Methods Appl. Mech. Engrg. 198 (2009) 2860-2873.

[29] P. Alart, A. Curnier, A mixed formulation for frictional contact problems prone to Newton like solution methods, Comput. Methods Appl. Mech. Engrg. 92 (1991) 353-375.

[30] M. Hintermüller, K. Ito, K. Kunisch, The primal-dual active set strategy as a semismooth Newton method, SIAM J. Optim. 13 (2003) 865-888.

[31] M. Gitterle, A. Popp, M.W. Gee, W.A. Wall, Finite deformation frictional mortar contact using a semi-smooth Newton method with consistent linearization, Int. J. Numer. Methods Engrg. 84 (2010) 543-571.

[32] S. Hüeber, B.I. Wohlmuth, Thermo-mechanical contact problems on nonmatching meshes, Comput. Methods Appl. Mech. Engrg. 198 (2009) 13381350.

[33] C. Hager, B.I. Wohlmuth, Nonlinear complementarity functions for plasticity problems with frictional contact, Comput. Methods Appl. Mech. Engrg. 198 (2009) 3411-3427.

[34] R. Krause, A nonsmooth multiscale method for solving frictional two-body contact problems in 2D and 3D with multigrid efficiency, SIAM J. Sci. Comput. 31 (2009) 1399-1423.

[35] İ. Temizer, P. Wriggers, T.J.R. Hughes, Three-dimensional mortar-based frictional contact treatment in isogeometric analysis with NURBS, Comput. Methods Appl. Mech. Engrg. 209-212 (2012) 15-128.

[36] L. De Lorenzis, I. Temizer, P. Wriggers, G. Zavarise, A large deformation frictional contact formulation using NURBS-based isogeometric analysis, Int. J. Numer. Methods Engrg. 87 (2011) 1278-1300.

[37] K. Washizu, Variational methods in elasticity and plasticity, Pergamon Press, London, 1968.

[38] J.C. Simo, Numerical analysis and simulation of plasticity, in: P.G. Ciarlet, J.L. Lions (Eds.), Handbook of Numerical Analysis, vol. VI, Elsevier, 1998.

[39] C. Hesch, P. Betsch, Transient three-dimensional contact problems: morta method. Mixed methods and conserving integration, Comput. Mech. 48 (2011) 461-475.

[40] A. Konyukhov, K. Schweizerhof, Covariant description for frictional contact problems, Comput. Mech. 35 (2005) 190-213. 
[41] I. Temizer, P. Wriggers, T.J.R. Hughes, Contact treatment in isogeometric analysis with NURBS, Comput. Methods Appl. Mech. Engrg. 200 (2011) 11001112.

[42] J. Lu, Isogeometric contact analysis: geometric basis and formulation of frictionless contact, Comput. Methods Appl. Mech. Engrg. 200 (2011) 726-741.

[43] J.-Y. Kim, S.-K. Youn, Isogeometric contact analysis using mortar method, Int. J. Numer. Methods Engrg. 89 (2012) 1559-1581.
[44] L. De Lorenzis, P. Wriggers, G. Zavarise, A mortar formulation for 3D large deformation contact using NURBS-based isogeometric analysis and the augmented Lagrangian method, Comput. Mech. 49 (2012) 1-20.

[45] D.A. Hills, D. Nowell, A. Sackfield, Mechanics of Elastic Contacts, Butterworth Heinemann, Oxford, 1993.

[46] C. Hesch, P. Betsch, Isogeometric analysis and domain decomposition methods, Comput. Methods Appl. Mech. Engrg. 213-216 (2012) 104-112. 